\title{
Spectral analysis of round jet instabilities at low Reynolds number
}

\author{
Najah Kechiche ${ }^{1, a}$, Ali Abbassi ${ }^{1}$, Taoufik Filali ${ }^{1}$, Jacques Jay ${ }^{2}$ and Habib Ben Aissia ${ }^{1}$ \\ 1 Unité de Métrologie en Mécanique des Fluides et Thermique, UMMFT 03/UR/11-09, Tunisie École Nationale d'Ingénieurs \\ de Monastir, Rue Ibn El Jazzar, 5019 Monastir, Tunisie \\ ${ }^{2}$ Hubert Curien Laboratory, Bâtiment F, 18 rue B. Lauras, 42000 St-Étienne, France
}

Received 23 March 2009, Accepted 7 December 2009

\begin{abstract}
For the study of round jet flow at low Reynolds number, a fine analysis of instabilities is necessary. In this paper, we present a frequency analysis of temporal and spatial velocity variations in a round jet with a Reynolds number equal to 830. The data were obtained from Laser Doppler Anemometry (LDA) measurements for various downstream positions. Attention is focused on the characteristics of the instantaneous signal of the longitudinal velocity component $u(t)$ in order to characterize the transition from laminar state to turbulent state. The spectral analysis of the LDA signals of the axial component of the exit velocities highlights the presence of a single peak of energy around a characteristic frequency $f_{0}$. This frequency corresponds to the amplified mode of the instability. The jet develops a sinuous mode which is the most unstable anti-symmetric mode. The results obtained in this study concerning the sinuous mode frequency, agree well with those obtained by laser tomography technique and images analysis.
\end{abstract}

Key words: Round jet / characteristic frequency / transition / sinuous mode / LDA / Fourier transform / laser tomography / Kelvin-Helmholtz instabilities / velocity profile

\begin{abstract}
Résumé - Analyse spectrale des instabilités d'un jet rond à bas nombre de Reynolds. Une analyse fine des instabilités est nécessaire pour l'étude de l'écoulement d'un jet à faible nombre de Reynolds. Dans cet article, nous présentons une analyse fréquentielle des variations de vitesse temporelle et spatiale dans un jet rond pour un nombre de Reynolds égale à 830. Les données ont été obtenues à partir des mesures par Anémométrie Laser à effet Doppler (ALD) pour différentes positions en aval. Une attention est donnée à la caractéristique du signal instantané de la composante longitudinale de vitesse $u(t)$ afin de caractériser la transition du laminaire au turbulent. L'analyse spectrale des signaux ALD de la composante axiale de la vitesse met en évidence la présence d'un seul pic d'énergie autour d'une fréquence $f_{0}$. Cette fréquence correspond au mode le plus amplifié de l'instabilité. Le jet développe un mode sinueux qui est le plus instable anti-symétrique mode. Les résultats de cette étude concernant la fréquence du mode sinueux, concordent avec ceux obtenus par la technique de tomographie laser et analyse d'images.
\end{abstract}

Mots clés : Jet rond / fréquence caractéristique / transition / mode sinueux / ALD / transformée de Fourier / tomographie laser / instabilités de Kelvin-Helmholtz / profil de vitesse

\section{Introduction}

Flow produced by round jet was the most intensively studied of all the classical shear layers like jets, mixing layer and wakes, due to its great interest from the perspective of aeronautical and potential industrial applications such as insulation by curtain of air, epitaxy using a carrier gas jet, heat insulation and so on. For engineering purposes, for example design of combustor burners, it is

a Corresponding author: kechiche2000@gmail.com of great interest to understand, in depth, the mechanism of transition from laminar to turbulence state. The transition to turbulence state is generally caused by the development and amplification of small disturbances from the surrounding ambient, generated typically by: vibrations of the mechanical structures, acoustic sources, etc. The purpose of this study is to encircle the essential mechanism within the framework of the hydrodynamic stability theory, and in particular, to obtain a criterion of selection of the oscillation frequency. It is found that, except at very low Reynolds numbers, a laminar thin shear layer 
with constant density is unstable to arbitrarily small disturbances. These disturbances evolve by selective amplification as they convect downstream [1]. The migration of vorticity resulting from the instability forms axisymmetric or helical structures accompanied by large amplitude fluctuations. Generally, these structures interact with one another and contribute significantly to entrainment and mixing by convective transport [2]; and to the generation of acoustic noise [3,4]. The linear stability theory describes, in a very satisfactory way, the initial instability development of the thin shear layer, which rolls up in discrete vortices of Kelvin-Helmholtz instabilities [5, 6]. Cohen and Wygnanski [7] showed that linear stability theory is able to predict details of the instability jet characteristics as the spectral distribution of the velocity perturbations. The varicose mode gives rise to axisymmetric, ring shaped vortices, whereas the amplification of the first helical mode $(m=1$, where $m$ is the azimuthal wave number) gives birth to helix vortices [8]. Linear analysis provides that, generally two modes of instabilities are observable in the free round jet, the varicose mode which is characterized by an axisymmetric development of the vortices (in the shape of rings) and the helical mode which corresponds to an antisymmetric configuration (progression in helix) [9].

In order to study the instability of an air jet, Boujemaa et al. [10] have performed an experimental study, using the LDA technique, to measure the velocity component at the jet exit. For their study, the Reynolds number is in the range $\left(500<R e_{D}<7000\right)$. They noticed that, for Reynolds numbers lower than 500, the flow was laminar even far from the nozzle exit (up to $30 D$ ). Beyond this limit, the helical mode becomes visible. However, they showed that the varicose mode starts to develop from approximately Reynolds number equal to 900 .

Zaouali et al. [11] have studied an incompressible, axisymmetric and downward free jet of air using the tomography Laser technique. In their study, these authors show that the laminar zone is being spread out over $17.5 \mathrm{D}$ for a Reynolds number $(R e=830)$. For Reynolds number equal to 1700 , the degeneration of the vortical structures starts at $6 D$ which made them notice that the varicose mode is the most unstable.

A direct numerical simulation study, using a temporal approach under the assumption that the studied instabilities are of convective nature, was made by Brancher [12]. This study showed that a weak excitation can start in the jet, which presents the oscillating type behaviour, an optimal response around a resonance frequency. The presence of self sustained oscillations in all the flow is the signature of a global mode [13].

A spectral analysis of the natural free plane jet realized by Meyer and Faghani $[14,15]$ showed that it is not very obvious to encircle the range of the excitations frequencies, due not only to the external disturbances, but also to those generated upstream jet emission. The examination of the power spectral density signals of the natural jet emission, showed that oscillations come from fluctuations at low frequencies (of frequencies average $8 \mathrm{~Hz}$ ) induced by the blower engine. The same authors and others underlined the difficulty of a frequential study of a natural jet in which the organization of vortical structures is intermittent. They also noted that the events which characterized the jet, such as vortex, rolling up or pairings, occur neither at fixed positions nor in a periodic way in time. Compared to the forced jet, the free jet thus constitutes a stage towards a flow less organized, which makes the analysis difficult. In the zone close to the free jet, as for the whole of the free sheared layers, initial mixing layers instabilities, develop until leading to vortex and rolling up at an abscissa $X_{\mathrm{s}}$ ( $X$ is the streamwise direction of the jet). This abscissa would mark the saturation of the fundamental instability with a frequency $f_{0}[2]$. In fact, this is the most amplified frequency by the jet in the unstable frequencies band. At the end of the potential zone, the frequency $f$ of the passage of vortical structures makes it possible to define the jet mode, whose relationship with the initial frequency instability of the mixing layer is not always simple [16].

In this paper, we propose an experimental study of the primary instability of an axisymmetric, downward and isotherm jet with initial uniform velocity profile (tophat). The experimental technique used to analyse the mechanism of the primary instability, which occurs in the transition region near to the exit of the nozzle, is the Laser Doppler Anemometry (LDA). A local instantaneous longitudinal velocity component is obtained and then analysed by means of Fourier transform. The results obtained from the spectral analysis of the velocity fluctuation evolution enabled us to determine the characteristic frequency of the sinuous mode, which is the most unstable mode in the primary transition region at Reynolds number equal to 830 . This experimental investigation is aimed at obtaining a clear physical understanding of the unstable modes which evolve in the near field (or the transition region) of round jets. We have chosen conditions for which the flow is incompressible and buoyancy effects are negligible with a long laminar zone as suggested by Ben Aissia [17].

\section{Experimental apparatus and procedures}

\subsection{Experimental arrangement}

Experiments were carried out on air into air axisymmetric free jet, which is generated by an axisymmetric nozzle of internal diameter equal to $12.4 \mathrm{~mm}$. The geometry of the nozzle was conceived so as to obtain a uniform profile velocity in the ejection section (top-hat). The jet velocity measurement was performed by using Laser Doppler Anemometry (LDA) in forward scatter. A schematic representation of the experimental device is given in (Fig. 1). The air coming from a tank passes through a filter with low porosity $(0.8 \mu \mathrm{m})$, its function is to remove the impurities, such as the water droplets because the presence of such particles can distort measurements by LDA technique. A valve punch placed downstream allows a manual fitting of the adjustment to 


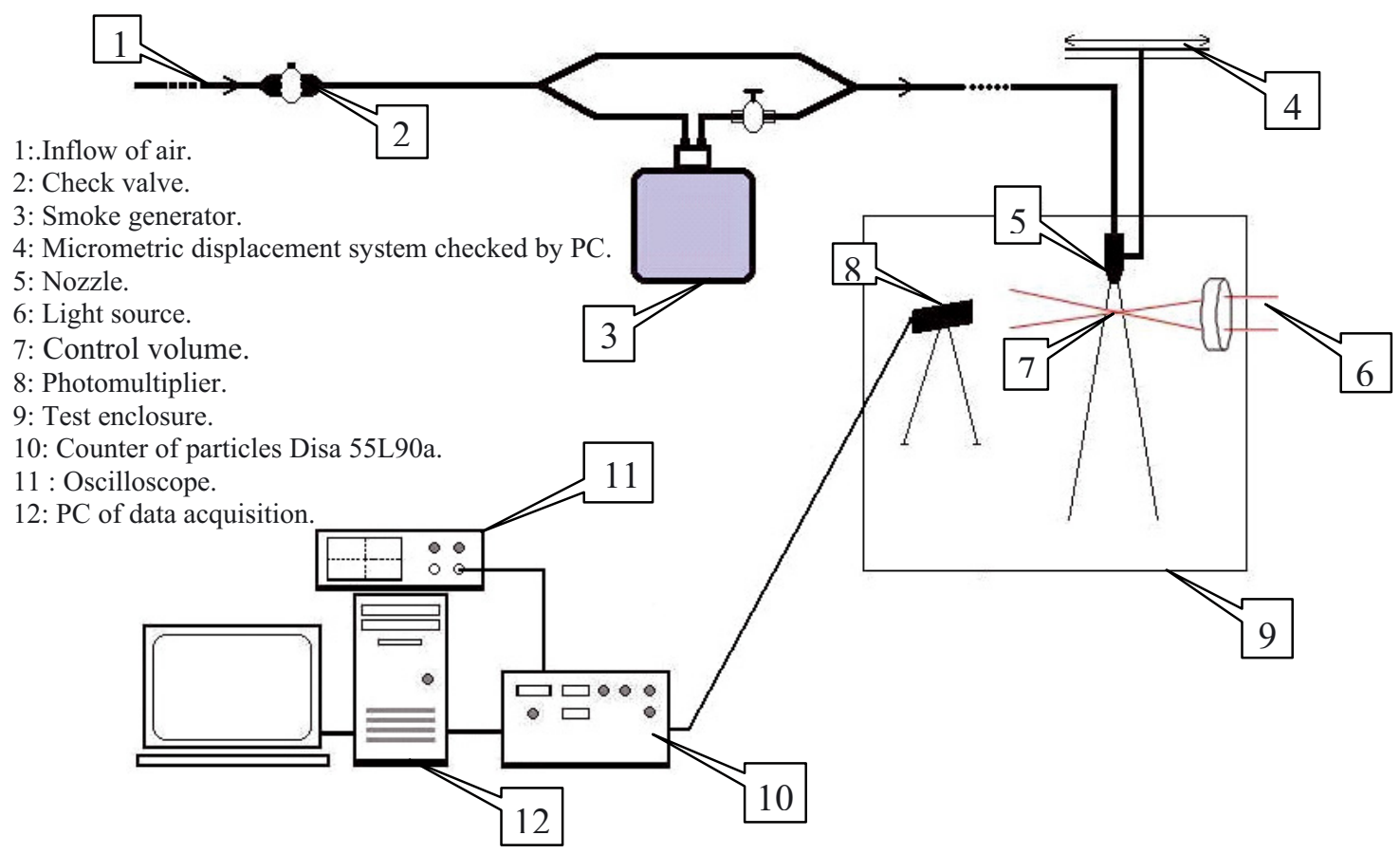

Fig. 1. Schematic of the measurement region and instrumentation.

regulate finely the flow rate, therefore a Reynolds number is well defined at the nozzle exit $R e=U_{0} D / \nu$, (where $\nu$ is the kinematic viscosity), based on the characteristic exit mean velocity $U_{0}$ and on the characteristic transverse dimension $D$ of the internal diameter of the nozzle. The flow crosses a smoke generator and before arriving at the exit of the nozzle, it passes through a plenum chamber. The latter is made up primarily of a honeycomb and screens which managed the jet flow quality, which produced a laminar flow at the exit of the nozzle. The nozzle is assembled vertically on a micrometric displacement system, which allows the positioning of the nozzle according to the vertical and horizontal directions, $x$ and $y$, respectively. The jet is injected vertically (downward), with the velocity value $1.04 \mathrm{~m} . \mathrm{s}^{-1}$ at the exit of the nozzle giving a Reynolds number equal to 830 in the present investigation. The seeding of the flow was by incense particles with a diameter varying between 0.5 and $5 \mu \mathrm{m}$. These particles are chosen in order to keep the fluid nature and are produced by a smoke generator [17]. Two parallel and coherent rays, resulting from the emission optics are focused at the measurement point: an interference phenomenon takes place with an interfrange equal to $2.85 \mu \mathrm{m}$. The particles which pass through the measurement point will reflect the light with a Doppler frequency proportional to their velocities. The reflected light is detected by a photomultiplier disposed in front of the measurement volume. The signal corresponding to each particle is called "burst". The frequency and other data, for example: period's number, passage times, time interval between each burst are given starting from the DISA 55L90a particles counter (DANTEC). A storage oscilloscope is used to obtain information on the output signal of the particles counter. An acquisition card was designed to acquire the data through a computer [18]. The whole system is enclosed in a Plexiglas cell with a volume of $6 \mathrm{~m}^{3}$ to isolate the jet from external disturbances. The temperature of the room is kept uniform by air conditioning. The obtained data will enable us to observe the principal frequencies at the centreline of the jet flow.

In this study, the data were obtained by Laser Doppler Anemometry (LDA) and only the axial $u$ velocity component has been considered. The signals from LDA were acquired at a sampling rate of $100 \mathrm{kHz}$. In each measurement, 4096 samples were acquired corresponding to signal duration of $40 \mathrm{~ms}$.

The velocity signals were obtained at one horizontal distance $r / D=0$ (where $r$ is the spanwise direction and $D$ is the internal diameter of the nozzle) and at several longitudinal distances $x / D$, where $x$ is the streamwise distance measured from the nozzle exit. We have used a non uniform step in the streamwise direction $x$ of the jet. At the exit of the nozzle we have used a step equal to $10 \mathrm{~mm}$, after the potential core we have increased the step to $20 \mathrm{~mm}$ to be able to go further downstream in the flow direction. The Fourier analysis has been widely used to characterize flows in which interacting modes are dominant [19].

\subsection{Inflow boundary conditions}

Before examining in depth the development and evolution of instabilities in the jet, it is necessary to define the mean characteristics of the initial shear layer. This description should include essentially the mean velocity and the intensity of turbulence which give the level of disturbances that exist in the initially laminar 

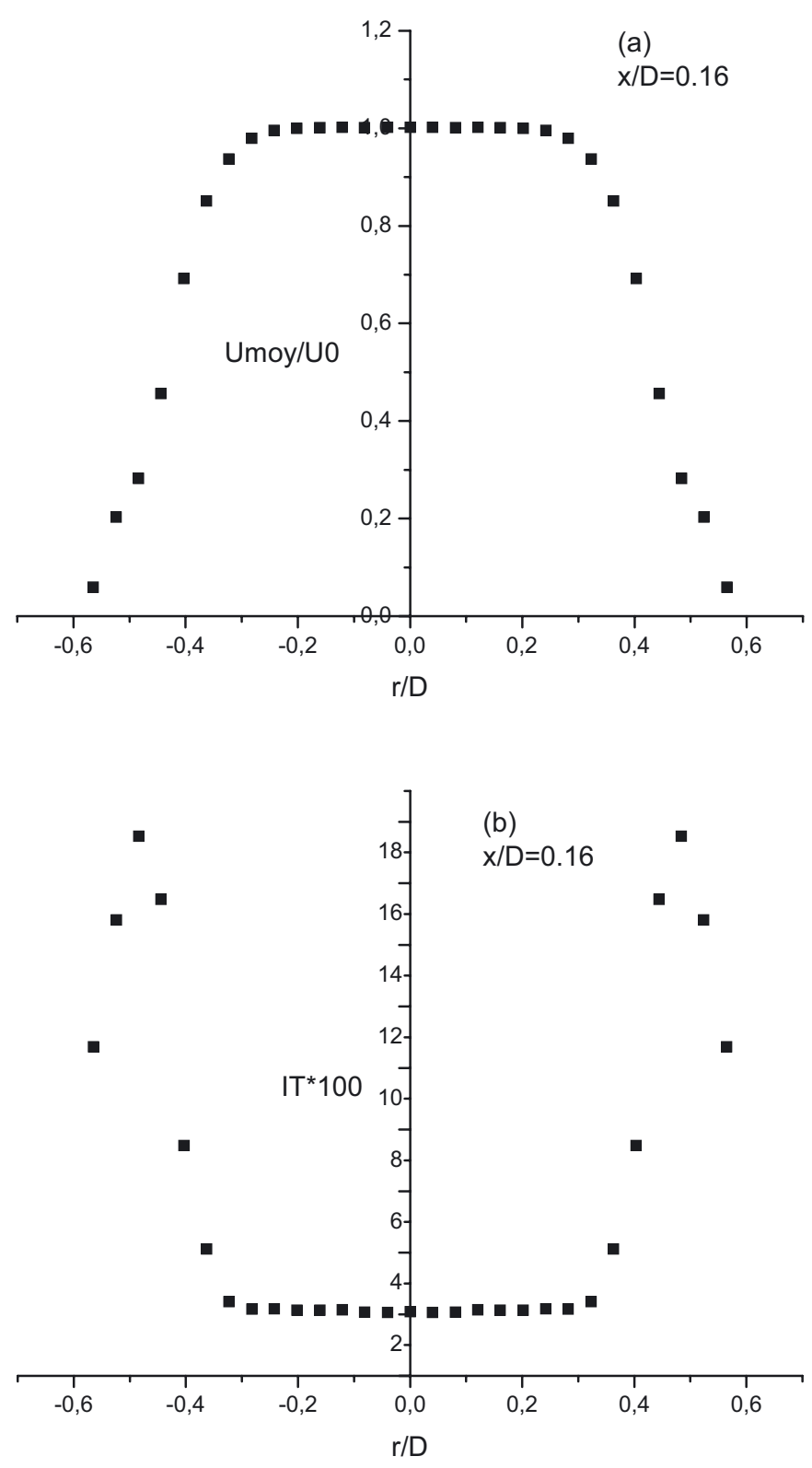

Fig. 2. (a) Inlet mean velocity profile. (b) Turbulence intensity profile.

boundary layer. These inflow boundary conditions are obtained starting from local measurements by LDA technique. Figures $2 \mathrm{a}, 2 \mathrm{~b}$ show the exit boundary-layer mean velocity $U / U_{0}$ and the longitudinal fluctuation intensity $u^{\prime} / U_{0}$ profiles, measured 0.16 downstream from the exit plane. The data in this figure were taken as close as possible to the nozzle exit using the LDA technique. Near the exit, the jet has a uniform (top-hat) profile as shown in Figure 2a. It should be noted that the mean velocity remains constant in the potential core region and decreases due to the increase in the rate of ambient gas that enters in the jet flow. The observation of the mean velocity profile let us consider that the first transition region behaves like a mixing layer $[6,21]$. The momentum thickness $\theta$ is

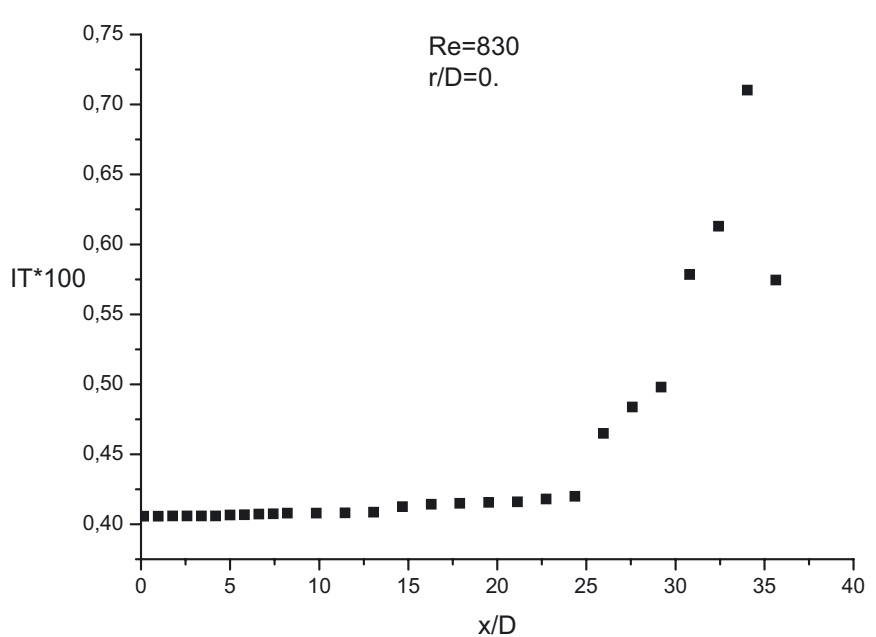

Fig. 3. Evolution of the axial turbulence intensity.

defined by $\theta=\int_{0}^{\infty} \frac{U}{U_{0}}\left(1-\frac{U}{U_{0}}\right) \mathrm{d} y$ and has a value of order $0.5 \mathrm{~mm}$ at the streamwise distance $x / D=0.16$ which gives for $R / \theta$ the value of 12.4 . The broadband turbulence, $u^{\prime} / U_{0}$ at this location of the flow $(x / D=0.16$, $r / D=0)$ is approximately $0.4 \%$ including instrument noise. The longitudinal velocity fluctuation intensity profiles $u^{\prime} / U_{0}$ (Fig. 2b) exhibit a peak at $r / D=0.484$, this being typical of jets with initially laminar shear layers [20]. Both the mean and fluctuating velocity data indicate that the initial boundary-layer at the exit of the nozzle is laminar for the Reynolds number 830 .

\section{Results and discussions}

For the jets, the transition from laminar to turbulence state takes place at some downstream distances from the nozzle. This can be seen more clearly, as shown in Figure 3, by observing the turbulence intensity, $\sqrt{\overline{u^{\prime 2}}} / U_{0}$, (where $\sqrt{\overline{u^{\prime 2}}}$ is defined as the rms longitudinal fluctuating velocity and $U_{0}$ is defined as the centreline velocity at the nozzle exit) at increasing downstream distances $x / D$ for Reynolds number equal to 830 . At $x / D$ of 3.38 and 9.84 the measured values of the turbulence intensities are approximately $0.4 \%$. Comparison between the intensities at $x / D$ of $30.8,32.42$ and 34 shows a gradual increase of turbulence intensity. The maximum of turbulence intensity of over $0.7 \%$ occurs at $x / D=34$ at the center of the jet. The production of turbulence by mean flow takes place in the high shear-region. The existence of the maximum at the centreline can only be attributed to large scalemixing across the jet. In short, for the Reynolds number equal to 830 used in the present investigation, the turbulence intensity increases, reaches a maximum value and then decreases. The location $x / D$ which corresponds to the rapid rise in the $\sqrt{\overline{u^{\prime 2}}} / U_{0}$ values may indicate transition from laminar to turbulence state. The value of the streamwise distance $x / D$ corresponding to maximum of 


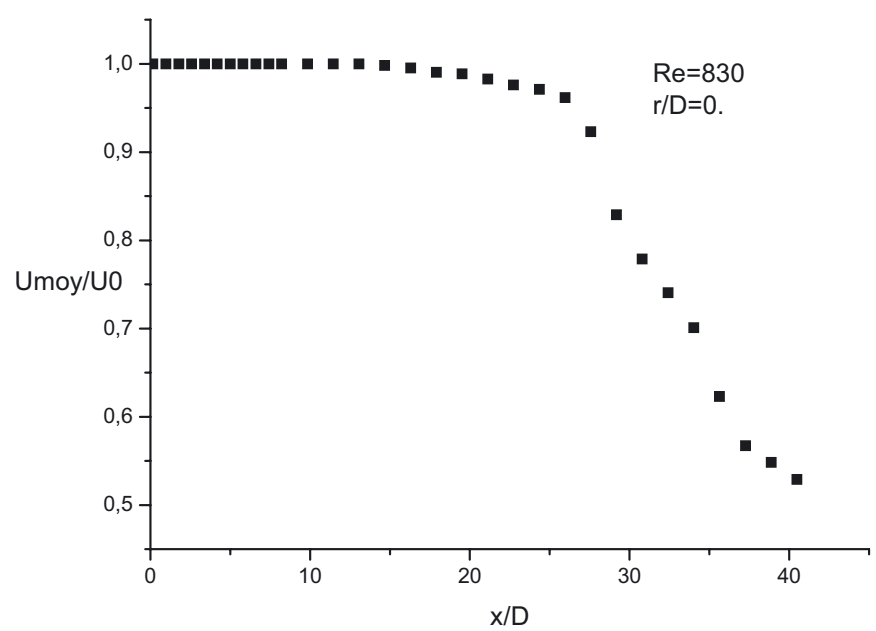

Fig. 4. Evolution of the centreline axial velocity.

turbulence intensity is of order $x / D=34$ in the present study as shown in Figure 3.

Figure 4 shows the centreline axial velocity, $U_{c}$, in the jet for the Reynolds number 830. Near the nozzle, the centreline velocity decreases only slightly with streamwise distances $x / D$ and in an approximately linear manner. Further downstream a location is reached where the centerline velocity decreases rapidly. The decrease in the centreline velocity is caused by the transition from laminar to turbulence state with high transfer of momentum. It defines another indicator to the transition region as well as the turbulence intensity.

We measured along the vertical axis of the jet a velocity value equal to $1.04 \pm 0.052 \mathrm{~m} . \mathrm{s}^{-1}$ for axial distances varying between $x=2 \mathrm{~mm}$ and $x=422 \mathrm{~mm}$ with an uncertainty value $\Delta x=0.01 \mathrm{~mm}$. The number of the FFT windows is 54 and 4096 samples per windows were taken at each measurement point.

The results are obtained by a spectral analysis carried out on the spectra of the longitudinal velocity fluctuation of the jet. These data are represented in a normalized scale in order to be able to follow the evolution of the characteristic frequency in the flow direction.

For a Reynolds number value equal to 830, the power spectral density (PSD) evolutions are represented in Figures $5 \mathrm{a}$ and $5 \mathrm{~b}$ for two longitudinal distances $x / D$ near the exit of the nozzle. As shown on (Fig. 5a), we notice the presence of several frequencies which have low values of PSD but they have the same order of magnitude. The jet is the result of a complex interaction between several modes which can grow or decay. At $x / D=9.8$ (Fig. 5b), for a frequency about $11 \mathrm{~Hz}$, a peak appears.

Figures $6 \mathrm{a}$ and $6 \mathrm{~b}$ show, for longitudinal positions $x / D$ equal to 17.9 and 25.9, respectively, the existence of a frequency peak which has an important value of PSD. But, at these distances, other peaks are present near to the maximal peak, this could be explained by the fact that several modes mix or by the instability of the one mode.
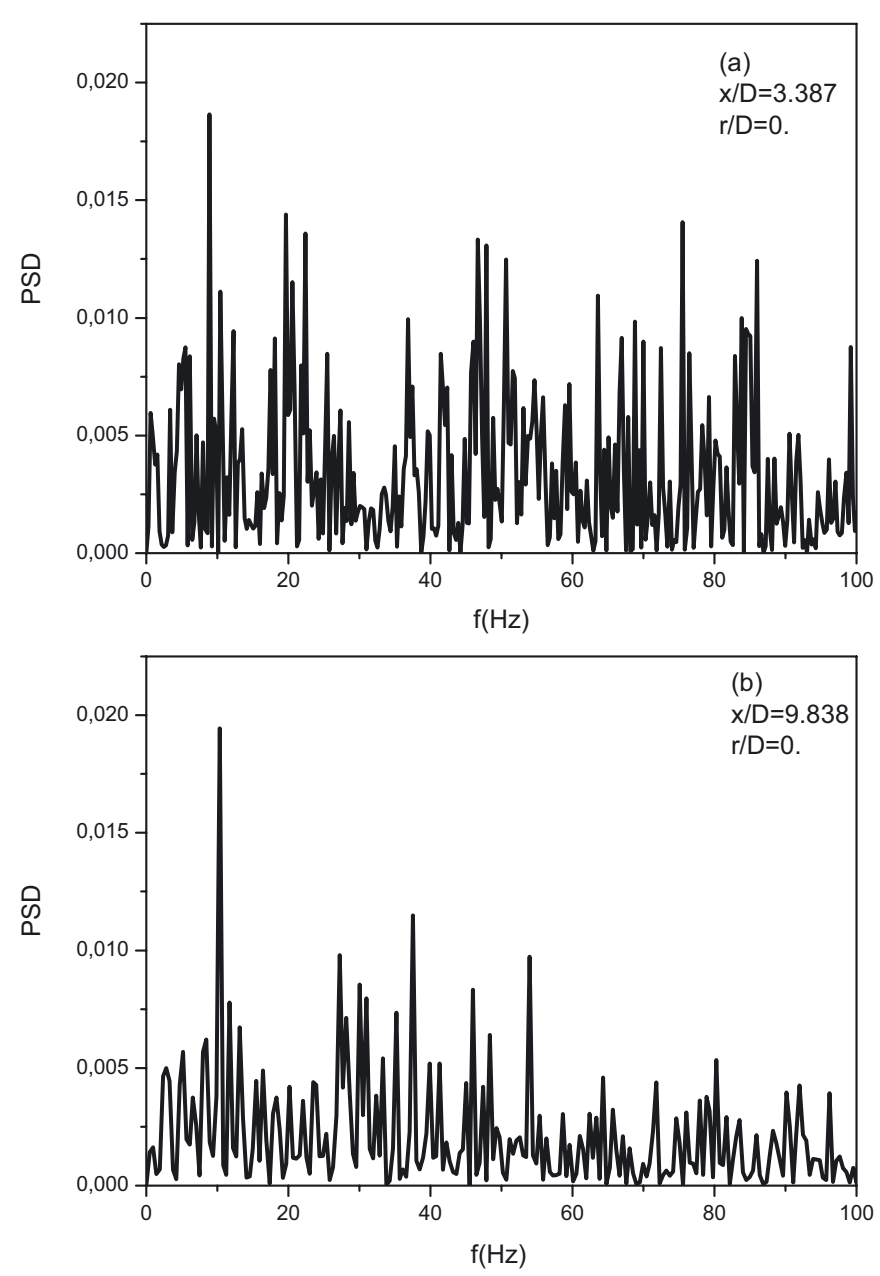

Fig. 5. Spectra of fluctuation velocity for $R e=830$, (a): $x / D=3.387$, (b): $x / D=9.838$.

For larger values of $x / D$, Figures $7 \mathrm{a}$ and $7 \mathrm{~b}$, we notice the presence of a characteristic frequency around $9 \mathrm{~Hz}$. For this frequency, the value of PSD is equal to 0.14 in the section $x / D=30.8$ (Fig. 7a) and grows to finally reach a maximum value equal to 7.8 for $x / D=34$ (Fig. 7b). We observe the presence of two peaks which appear towards $x / D=17$ and persist up to $30 \mathrm{D}$ with better definition at the distance $x / D=34$. A third peak at weaker frequency appears downstream i.e. with $x / D=30$ and seems to develop towards $x / D=34$.

It should be mentioned that the last position $x / D=$ 34 is particular because it corresponds to the zone in which the mean velocity falls quickly and the value of the turbulence intensity increases considerably (Fig. 3).

In fact, other instabilities are born at this position $x / D=34$ (secondary instabilities). It is noticed that the other frequencies which were present in the jet at the region close to the exit of the nozzle have a low amplitude compared to that of the most unstable jet mode at the longitudinal distance $x / D=34$. For this Reynolds, the most unstable mode is the sinuous mode.

As the origin of natural perturbations is upstream of the jet exit, the spectral analysis of the LDA signals of 

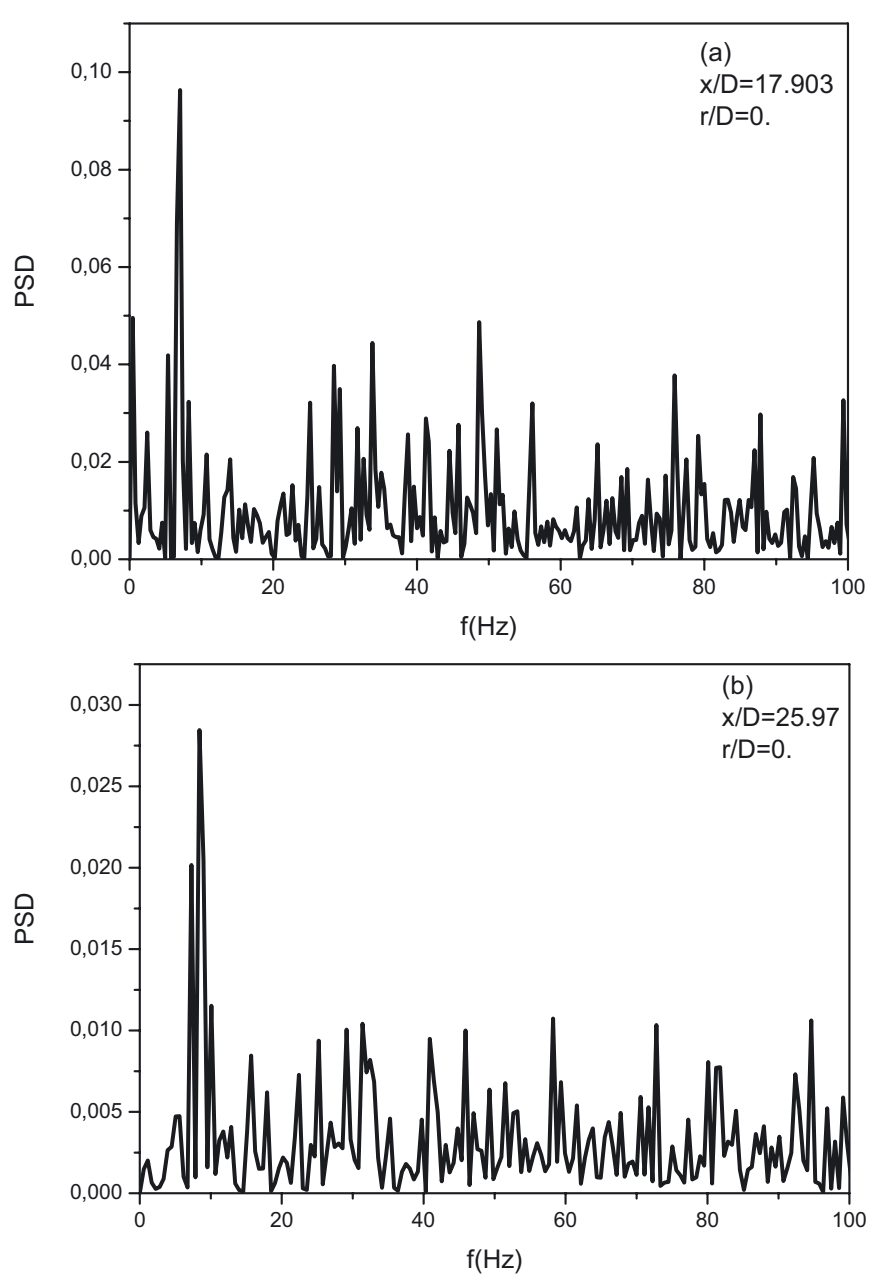

Fig. 6. Spectra of fluctuation velocity for $R e=830$, (a): $x / D=17.903,(\mathrm{~b}): x / D=25.97$.

the axial component of the exit velocities highlights the presence of a single very fine peak of energy around a characteristic frequency $f_{0}$.

Figure 8 shows the evolution of the jet frequency of the amplified mode at several $x / D$ downstream the exit of the nozzle. The variation of the frequency according to $x / D$ shows that this last parameter keeps an approximately constant value; this is explained by the presence of the sinuous mode characterized by the absence of pairing. This frequency is detectable in the initial zone near to the exit of the jet. When the value of the PSD becomes maximal, the jet selects a frequency value. The characteristic frequency of the jet is determined by the geometry and the velocity of the flow. The initial conditions at the exit of the nozzle are kept constant ( $U_{0}$ and $D$ are constant) corresponding to a Strouhal number $S t=0.11$.

In Figure 9 we have represented the evolution of the PSD according to $x / D$ and we note that in the initial zone (potential core) the flow is slightly disturbed. The comparison between the frequencies values at the potential core shows that the noise and the fluctuation of vortical structures have the same energy. Downstream and far from the exit of the nozzle, the vortical structures are
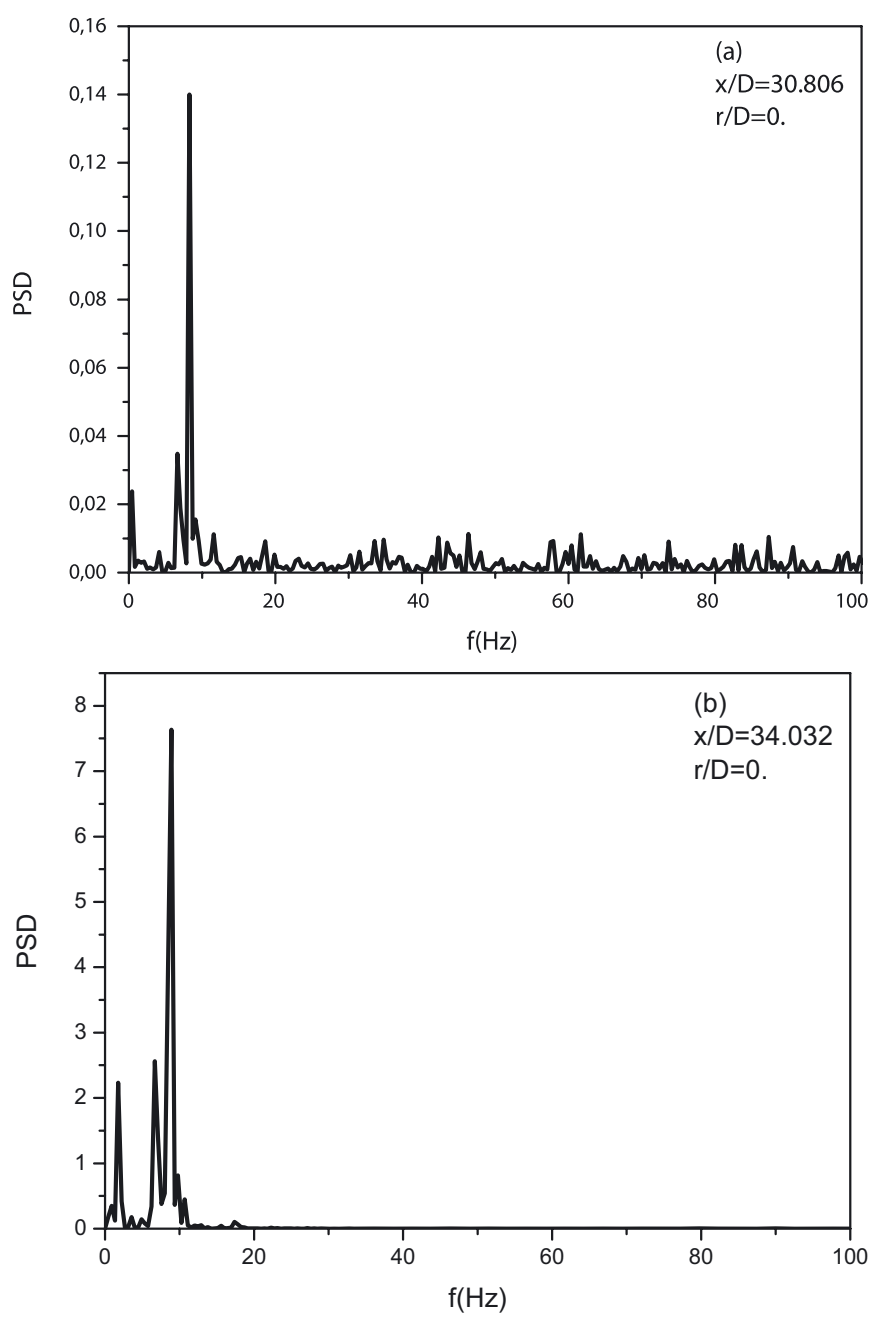

Fig. 7. Spectra of fluctuation velocity for $R e=830$, (a): $x / D=30.806,(\mathrm{~b}): x / D=34.032$.

highlighted by the velocity measurement and the value of PSD is larger compared to the other fluctuations. Upstream from the exit of the nozzle, there is a package of disturbance which will be conveyed by the jet and progressively (more and more downstream from the exit of the nozzle) the jet will amplify the principal disturbance which is characterized by a dominant fundamental frequency. If one represents the evolution of the intensity of the turbulence according to $x / D$ for the Reynolds number equal to 830 (Fig. 3), one notices that it increases which explains the peak of frequency of the spectrum which increases in the same way [21].

In order to compare our results with those obtained using the Laser Tomography measurement technique, the sinuous zone is located and extracted from an acquired image (Fig. 10). The extracted part presents two antisymmetric boundaries in sinusoidal form. Each boundary presents convex parts (creast) and concave parts (trough) [22]. Indeed, the results of the jet profiles velocity, obtained using the Laser Tomography technique, [12] keep a constant value for the longitudinal positions varying between $x / D=20$ and $x / D=25$. The phase 


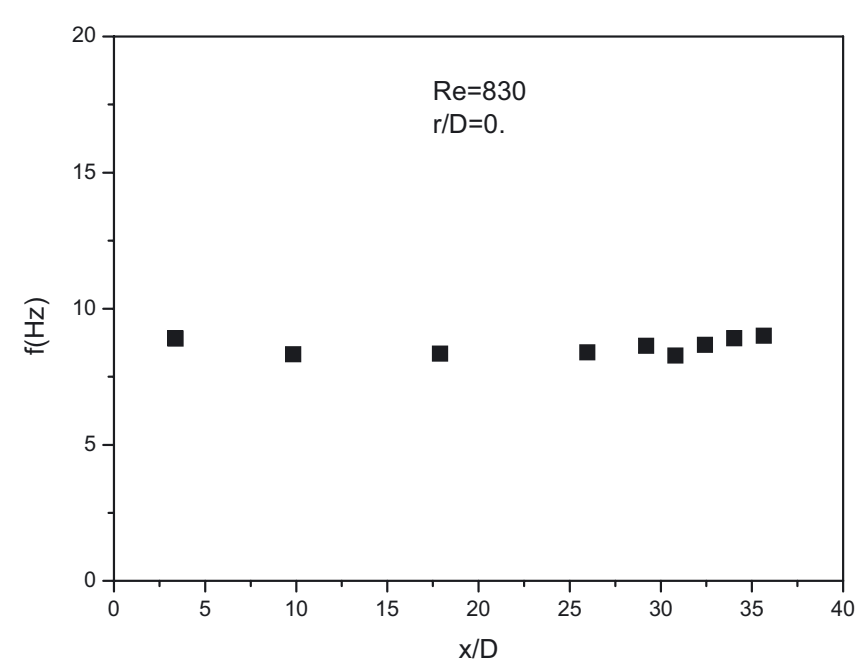

Fig. 8. Evolution of the characteristic frequency.

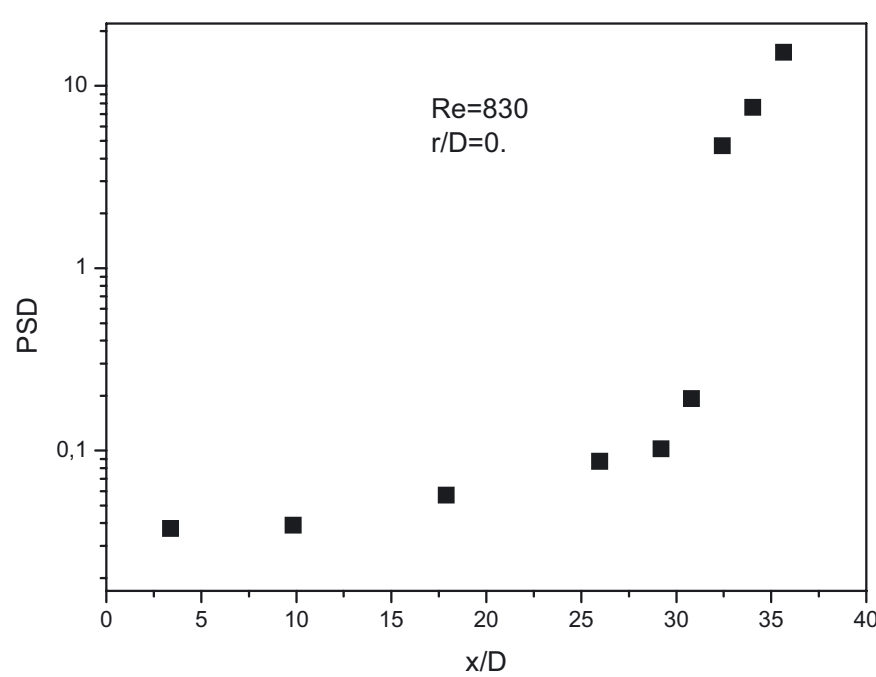

Fig. 9. Evolution of the Power Spectral Density (PSD).

velocity is about $0.46 \mathrm{~m} . \mathrm{s}^{-1}$ with an uncertainty value 0.08 and the average wavelength is approximately $54 \mathrm{~mm}$ for a Reynolds number equal to 830 . The characteristic frequency obtained for the sinuous instabilities is about $8.54 \mathrm{~Hz}$. This value is close to that found in the present study.

The difference between the frequency found by the measurements made by using the LDA and that obtained starting from the images by laser tomography is $0.4 \%$. The information in time given by the LDA technique allows the capture of instabilities that the tomography does not make it possible to see them (appearance of the secondary peaks).

On one hand, the LDA technique measurement proves the existence of many modes of instabilities in the jet since we noticed the presence of several frequencies, on the other hand, the Laser Tomography measurement is limited and showed only the amplified instabilities modes in the jet study.

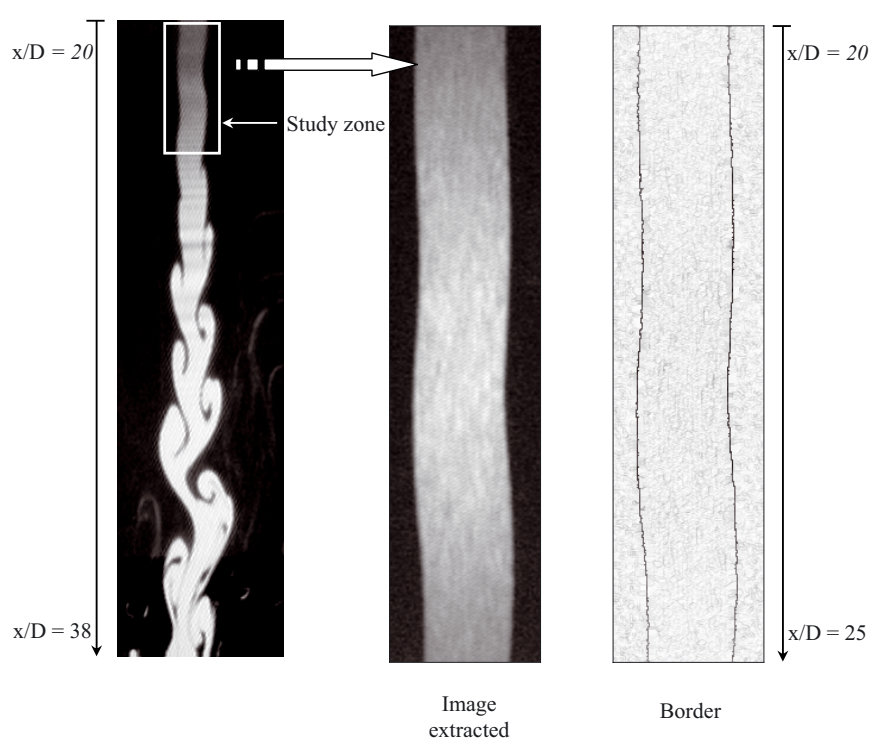

Fig. 10. Description of the sinuous mode (Zaouali et al. [22]).

\section{Conclusion}

The flow field structures in the central region of the round jet flow were analyzed with the LDA experimental technique. The LDA measurement of the streamwise velocity signal and the Fourier analysis of the frequency of the dominant mode have been carried out. This mode is the most unstable (called helical mode) which occurs in the first shear layer region of the jet.

The value of the power spectral density (PSD) is low for streamwise distances close to the exit of the nozzle. At these distances, the existence of several frequencies is noted. For further longitudinal distances, it becomes very significant for only one frequency which represents the most energetic mode in the jet. The characteristic frequency obtained from this analysis has a value around $9 \mathrm{~Hz}$. A comparison between our results and that obtained using the Laser Tomography [22] (by determining the phase velocity and the average wavelength) was carried out. Both results obtained for the frequency of the primary transition in the round jet, caused by the antisymmetric mode (sinuous mode), show a satisfactory agreement. The LDA makes it possible to measure the frequencies characteristic of the jet in the sinuous zone with good precision compared to the laser tomography. It is interesting to note that for high velocities (high Reynolds numbers), the LDA technique gives results more precise than those given by the Laser Tomography technique. That opens new possibilities for the analysis of primary and also secondary instabilities. The Laser Tomography does not visualize the other frequencies which can exist in the jet, because its study is limited for a well-defined length and we can not detect all the instabilities modes. Moreover, the visualization time is slow and the flow at this time takes other forms and changes its state that causes the loss of information. On the other hand, the LDA allows local measurements; and the spectra velocities show the existence of several frequencies other than 
the characteristic frequency. To obtain better information on the development of instabilities, it would be interesting to measure apart from the axis i.e. over the width of the jet. We can also study the instabilities for other Reynolds numbers to see the effect of this parameter on the development of instabilities in order to determine its nature (convective or absolute).

\section{References}

[1] F. Hussain, K. Zaman, An Experiment study of organized motions in the turbulent plane mixing layer, J. Fluid Mech. 159 (1985) 85-104

[2] C.D. Winant, F.K. Browand, Vortex pairing: the mechanism of turbulent mixing-layer growth at moderate Reynolds numbers, J. Fluid Mech. 63 (1974) 237-255

[3] V. Kibens, Discrete noise spectrum generated by an acoustically excited jet, AIAA J. 18 (1980) 434-441

[4] D.G. Crighton, Basic principles of aerodynamic noise generation, Prog. Aero. Sci. 16 (1975) 31-96

[5] A. Michalke, Survey on jet instability theory, Prog. Aero. Sci. 21 (1984)

[6] C.M. Ho, P. Huerre, Perturbed Shear Layers, Ann. Rev. Fluid Mech. 16 (1984) 365-424

[7] J. Cohen, I. Wygnanski, The evolution of instabilities in the axisymmetric jet, Part 1 . The linear growth of disturbances near nozzle, J. Fluid Mech. 176 (1987) 191-219

[8] G.K. Batchelor, A.E. Gill, Analysis of the Stability of Axisymmetric Jets, J. Fluid Mech. 14 (1962) 529-551

[9] I. Danaila, Étude des instabilités et des structures cohérentes dans la zone de proche sortie d'un jet axisymétrique, Thèse de doctorat, Univ. de la Méditerranée Aix-Marseille II, 1997

[10] S. Boujemaa, M. Aamielh, M.P. Chauve, Analyse spatiotemporelle de jets axisymétriques d'air et d'helium, C. R. Mécanique, Académie des sciences 332 (2004) 933-939

[11] Y. Zaouali, Étude tomographique des instabilités d'un jet axisymétrique à bas nombre de Reynolds par PIV et analyse des frontières, Thèse de doctorat, Univ. de centre Tunisie, 2004
[12] P. Brancher, Étude numérique des instabilités secondaires de jets, Thèse de doctorat, École Polytechnique, Laboratoire d'Hydrodynamique, 1996

[13] S. Ravier, Étude des instabilités d'un jet plan à masse volumique variable, Thèse de doctorat, Université de la méditerranée-Aix Marseille II, 2004

[14] J. Meyer, Structures organisées et transition dans la zone proche du jet plan : synthèse d'analyse expérimentale visuelle et numérique, Thèse de doctorat de spécialité, Institut national polytechnique de Toulouse, 1989

[15] D. Faghani, Étude des structures tourbillonnaires de la zone proche d'un jet plan : approche non stationnaire multidimensionnelle, Thèse de doctorat de spécialité, Institut national polytechnique de Toulouse, 1996

[16] E. Gutmark, C.-M. Ho, Preferred modes and the spreading rates of jets, Phys. Fluids. 26 (1983) 2932-2938

[17] H. Ben Aissia, Étude numérique et expérimentale par imagerie et anémométrie laser doppler d'un jet axisymétrique, Docteur Es Sciences Physiques, Université El Manar II, Tunisie, 2002

[18] N. Filali, N. Kechiche, T. Filali, H. Ben Aissia, Système d'acquisition temps réel pour l'anémométrie laser à effet Doppler, $7^{\mathrm{e}}$ colloque de recherche en physique, 2124 décembre 2003, Hammamet, Tunisie

[19] T. Yilmatz, A. Kodal, An analysis on coaxial jet flows using different decomposition techniques, J. Fluid Structures 14 (2000) 359-373

[20] M. Abid, M.E. Brachet, Numerical characterization of the dynamic of vortex filaments in round jets, Phys. Fluids 5 (1993) 2582

[21] A.K. Hussain, M.F. Zedan, Effects of the initial condition of the axisymmetric free shear layer: Effects of the initial momentum thickness, Phys. Fluids 21 (1978) 1100-1111

[22] Y. Zaouali, H. Ben Aissia, N. Kechiche, J. Jay, J-P. Schon, Experimental study of the instabilities in the laminar turbulent transition zone of an axisymmetric jet at low Reynolds number, J. Flow Visualization and Image Processing 11 (2004) 1-16 